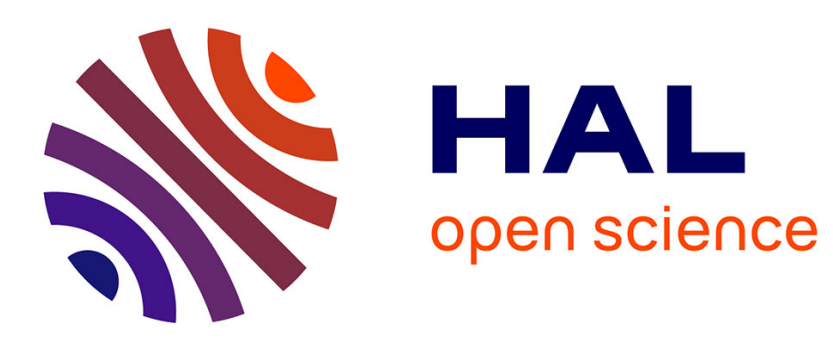

\title{
L'hypothèse de la polarisation du vide en électromagnétisme classique \\ Émile Durand
}

\section{To cite this version:}

Émile Durand. L'hypothèse de la polarisation du vide en électromagnétisme classique. J. Phys.

Radium, 1955, 16 (4), pp.262-270. 10.1051/jphysrad:01955001604026201 . jpa-00235141

\section{HAL Id: jpa-00235141 \\ https://hal.science/jpa-00235141}

Submitted on 1 Jan 1955

HAL is a multi-disciplinary open access archive for the deposit and dissemination of scientific research documents, whether they are published or not. The documents may come from teaching and research institutions in France or abroad, or from public or private research centers.
L'archive ouverte pluridisciplinaire HAL, est destinée au dépôt et à la diffusion de documents scientifiques de niveau recherche, publiés ou non, émanant des établissements d'enseignement et de recherche français ou étrangers, des laboratoires publics ou privés. 


\title{
L'HYPOTHĖSE DE LA POLARISATION DU VIDE EN ÉLEGTROMAGNÉTISME GLASSIQUE
}

\author{
Par ÉmILE DURAND,
}

Faculté des Sciences de Toulouse, Physique théorique.

Sommaire. - On montre qu'il est possible de considérer le vide comme un milieu polarisé. En tout point où le champ électrique est $\vec{E}$ et l'induction magnétique $\vec{B}$, les densités de polarisation électrique $\vec{P}$ et magnétique $\vec{M}$ ont pour expression

$$
\vec{P}=-\varepsilon_{0} \vec{E}, \quad \vec{M}=\frac{\vec{B}}{\mu_{0}} .
$$

Dans les milieux matériels isotropes, on remplace simplement $\varepsilon_{0}, \mu_{0}$ par $\varepsilon, \mu$.

On est ainsi conduit à une formulation un peu différente de l'électromagnétisme sans modifier en rien les grandeurs mesurables. En particulier, on est amené à définir un potentiel de polarisation pour une charge ponctuelle au repos ou en mouvement, pour un courant linéaire, etc. et ces potentiels ont un véritable sens physique. Ce ne sont pas de simples artifices de calcul, comme le feuillet polarisé normalement ou la courbe polarisée longitudinalement, qui ont été étudiés ailleurs par l'auteur [1], [2].

1. Rappel des formules concernant la polarisation des milieux matériels [3]. - Considérons un volume $v$ polarisé, limité par une surface $S$, dans lequel n'existe ni charges ni courants. Ce milieu est caractérisé par une densité de polarisation électrique $\vec{P}(x, y, z, t)$ et une densité de polarisation magnétique $\vec{M}(x, y, z, t)$. Les potentiels de polarisation électrique et magnétique sont définis par les expressions

$$
\left.\begin{array}{l}
\overrightarrow{\mathrm{II}}=\frac{\mathrm{I}}{4 \pi} \int_{v}[\vec{P}] \frac{\mathrm{d} v}{r}, \\
\vec{\Theta}=\frac{\mu_{0}}{4 \pi} \int_{v}[\vec{M}] \frac{\mathrm{d} v}{r},
\end{array}\right\}
$$

où les crochets désignent les fonctions prises au temps retardé $t-\frac{r}{c}$. Ces densités $\vec{P}$ et $\vec{M}$ sont définies à partir des charges et des courants électriques; on peut aussi considérer les densités $\vec{M}^{*}$ et $\vec{P}^{*}$ définies à partir des charges et courants magnétiques, qui sont reliées aux précédentes par les relations

$$
\vec{M}^{\star}=\frac{\vec{P}}{\varepsilon_{0}}, \quad \vec{P}^{\star}=\mu_{0} \vec{M}
$$

Ces potentiels de polarisation servent à définir les potentiels et antipotentiels classiques par les 
expressions

$$
\left.\begin{array}{cc}
\vec{A}=\mu_{0} \partial_{t} \vec{\Pi}+\overrightarrow{\operatorname{rot}} \overrightarrow{\boldsymbol{\theta}}, \quad & \vec{A}^{\star}=\varepsilon_{0} \partial_{t} \overrightarrow{\boldsymbol{\theta}}-\overrightarrow{\operatorname{rot}} \vec{\Pi}, \\
\varepsilon_{0} I=-\operatorname{div} \vec{\Pi} ; & \mu_{0} \cdot V^{\star}=-\operatorname{div} \overrightarrow{\boldsymbol{\theta}} .
\end{array}\right\}
$$

En portant (1) dans (2) et en transformant certaines intégrales de volume en intégrales de surface, on obtient

$$
\begin{aligned}
& \vec{A}=\frac{\mu_{0}}{4 \pi}\left\{\int_{v}\left[\overrightarrow{i^{\prime}}\right] \frac{\mathrm{d} v}{r}+\int_{v}\left[\overrightarrow{k^{\prime}}\right] \frac{\mathrm{d} S}{r}\right\}, \\
& V=\frac{\mathrm{I}}{4 \pi \varepsilon_{0}}\left\{\int_{v}\left[\rho^{\prime}\right] \frac{\mathrm{d} v}{r}+\int_{v}\left[\sigma^{\prime}\right] \frac{\mathrm{d} S}{r}\right\}, \\
& \vec{A}^{\star}=\frac{\varepsilon_{0}}{4 \pi}\left\{\int_{v}\left[\overrightarrow{i^{\star}}\right] \frac{\mathrm{d} v}{r}+\int_{v}\left[\overrightarrow{k^{\star}}\right] \frac{\mathrm{d} S}{r}\right\}, \\
& V^{\star}=\frac{\mathrm{I}}{4 \pi \mu_{0}}\left\{\int_{v}\left[\rho^{\star}\right] \frac{\mathrm{d} v}{r}+\int_{v}\left[\sigma^{\star}\right] \frac{\mathrm{d} S}{r}\right\},
\end{aligned}
$$

à condition de poser

$$
\begin{gathered}
\overrightarrow{i^{\prime}}=\partial_{t} \vec{P}+\overrightarrow{\operatorname{rot}} \vec{M}, \\
\rho^{\prime}=-\operatorname{div} \vec{P}, \\
\overrightarrow{k^{\prime}}=-[\vec{n} \times \vec{M}], \\
\sigma^{\prime}=(\vec{n} \cdot \vec{P}) ; \\
\overrightarrow{i^{\star}}=\mu_{0} \partial_{t} \vec{M}-\varepsilon_{0}^{-1} \overrightarrow{\operatorname{rot}} \vec{P}=\partial_{t} \vec{P}^{\star}-\overrightarrow{\operatorname{rot}} \vec{M}^{\star}, \\
\rho^{\star}=-\mu_{0} \operatorname{div} \vec{M}=-\operatorname{div} P^{\star} \\
\vec{k}^{\star}=\varepsilon_{0}^{-1}[\vec{n} \times \vec{P}]=\left[\vec{n} \times \vec{M}^{\star}\right], \\
\sigma^{\star}=\mu_{0}(\vec{n} \cdot \vec{M})=\left(\vec{n} \cdot \overrightarrow{P^{\star}}\right) .
\end{gathered}
$$

On définit ainsi les densités fictives de courantscharges électriques et magnétiques.

Des définitions (1) et (2), on en déduit les relations

$$
\begin{aligned}
& \underset{0}{1 / 2}\} \mu_{0} \vec{M}=\mu_{0}\left(\overrightarrow{\operatorname{grad}} V^{\star}+\partial_{t} \overrightarrow{A^{\star}}\right)+\overrightarrow{\operatorname{rot}} \vec{A}, \\
& \left.\left.\begin{array}{c}
1 / 2 \\
0
\end{array}\right\} \quad \vec{F}=\varepsilon_{0}\left(\overrightarrow{\operatorname{grad}} V+\partial_{t} \vec{A}\right)-\overrightarrow{\operatorname{rot}} \vec{A}^{\star} .\right\}
\end{aligned}
$$

Les champs et les inductions sont définis à partir des potentiels et antipotentiels par (1)

$$
\begin{aligned}
& \vec{E}=-\overrightarrow{\operatorname{grad}} V-\partial_{t} \vec{A}, \\
& \vec{B}=\overrightarrow{\operatorname{rot}} \vec{A} \\
& \overrightarrow{B^{\star}}=-\overrightarrow{\operatorname{rot}} \vec{A}^{\star} \\
& \overrightarrow{E^{\star}}=-\overrightarrow{\operatorname{grad}} V^{\star}-\partial_{t} \vec{A}^{\star} \text {. }
\end{aligned}
$$

(1) On désigne par $\vec{B}^{*}$ et $\vec{E}^{*}$ l'induction électrique et le champ magnétique qui sont habituellement représentés par les lettres $\vec{D}$ et $\vec{H}$. De cette manière toutes les grandeurs associées aux masses magnétiques sont affectées d'un astérique et l'on a la correspondance $\vec{P} \rightarrow \vec{P}^{*}, \vec{M} \rightarrow \vec{M}^{*}, \vec{E} \rightarrow \vec{E}^{*}$, $\vec{B} \rightarrow \vec{B}^{*}, \ldots$
En portant ces expressions (8), (9) dans (7), on trouve les relations classiques

$$
\vec{B}=\mu_{0}\left(\vec{E}^{\star}+\vec{M}\left(\begin{array}{c}
1 \\
1 / 2 \\
0
\end{array}\right), \quad \vec{E}=\varepsilon_{0}^{-1}\left(\vec{B}^{\star}-\vec{P}\left(\begin{array}{c}
1 \\
1 / 2 \\
0
\end{array}\right) .\right.\right.
$$

De (8), (9) on en déduit que l'on a identiquement

$$
\left.\begin{array}{rlrl}
\operatorname{rot} \vec{E}+\partial_{t} \vec{B} & =0, & \operatorname{rot} \vec{E}^{\star}-\partial_{t} \vec{B}^{\star} & =o, \\
\operatorname{div} \vec{B} & =0 ; & \operatorname{div} \vec{B}^{\star}=o .
\end{array}\right\}
$$

Ce sont les équations de Maxwell. Si l'on ne conserve que les deux vecteurs $\vec{E}, \vec{B}$, elles s'écrivent

$$
\left.\begin{array}{rl}
\operatorname{rot} \vec{E}+\partial_{t} \vec{B} & =0, \\
\operatorname{div} \vec{B} & =0 ; \\
\overrightarrow{\operatorname{rot}} \vec{B}-\varepsilon_{0} \mu_{0} \partial_{t} \vec{E} & =\mu_{0} \overrightarrow{\vec{i}^{\prime}}, \\
\operatorname{div} \vec{E} & =\frac{\rho^{\prime}}{\varepsilon_{0}},
\end{array}\right\}
$$

et si l'on ne conserve que les deux vecteurs $\vec{B}^{*}$, $\vec{E}^{*}$, elles prennent la forme

$$
\begin{aligned}
\overrightarrow{\operatorname{rot}} \vec{B}^{\star}+\varepsilon_{0} \mu_{0} \partial_{t} \vec{E}^{\star} & =-\varepsilon_{0} \overrightarrow{i^{\star}} \\
\operatorname{div} E^{\star} & =\frac{\rho^{\star}}{\mu_{0}} \\
\overrightarrow{\operatorname{rot}} \vec{E}^{\star}-\partial_{t} \vec{B}^{\star} & =o \\
\operatorname{div} \vec{B}^{\star} & =0
\end{aligned}
$$

2. L'hypothèse de la polarisation du vide en l'absence de polarisation matérielle. - Dans ce cas, on a

$$
\vec{B}=\mu_{0} \vec{E}^{\star}, \quad \varepsilon_{0} \vec{E}=\vec{B}^{\star}
$$

et les équations de Maxwell s'écrivent

$$
\left.\begin{array}{rl}
\operatorname{rot} \vec{E}+\partial_{t} \vec{B} & =0, \\
\operatorname{div} \vec{B} & =0 ; \\
\overrightarrow{\operatorname{rot}} \vec{B}-\varepsilon_{0} \mu_{0} \partial_{\iota} \vec{E} & =\mu_{0} \vec{i}, \\
\operatorname{div} \vec{E} & =\frac{\rho}{\varepsilon_{0}}
\end{array}\right\}
$$

Les densités singulières (ponctuelles, linéaires, superficielles) n'apparaissent pas dans les équations de Maxwell, à moins qu'elles ne figurent aussi dans $\vec{i}$, ... Mais elles se manifestent par des valeurs infinies ou des dicontinuités du champ. Par exemple, dans le cas des distributions superficielles, elles apparaissent dans les conditions aux limites

$$
\vec{B}_{(+)}-\vec{B}_{(-)}=\mu_{0}[\vec{k} \times \vec{n}], \quad \vec{E}_{(+)}-\vec{E}_{(-)}=\frac{\sigma}{\varepsilon_{0}} \vec{n}
$$


$\vec{n}$ étant le vecteur unitaire de la normale positive à la surface.

Les potentiels correspondant aux distributions volumiques s'écrivent

$$
\left.\begin{array}{c}
\vec{A}=\frac{\mu_{0}}{4 \pi} \int_{v}[\vec{i}] \frac{\mathrm{d} v}{r}, \quad V=\frac{\mathrm{I}}{4 \pi \varepsilon_{0}} \int_{v}[\rho] \frac{\mathrm{d} v}{r}, \\
\vec{A}^{\star}=V^{\star}=0
\end{array}\right\}
$$

et ceux des distributions superficielles ont pour expressions

$$
\left.\begin{array}{c}
\vec{A}=\frac{\mu_{0}}{4 \pi} \int_{S}[\vec{k}] \frac{\mathrm{d} S}{r}, \quad V=\frac{\mathrm{I}}{1 \pi \varepsilon_{0}} \int_{S}[\sigma] \frac{\mathrm{d} S}{r}, \\
\vec{A}^{\star}=I^{\star}=0 .
\end{array}\right\}
$$

On aurait des expressions analogues pour des distributions linéaires et ponctuelles. Les vecteurs de champ obéissant à (15), (16) se déduisent de (17), (18) par les expressions (8), (9).

Nous allons montrer que, dans ces conditions, il est possible de considérer le vide ou les régions où se trouvent les charges, comme un milieu polarisé dont les densités de polarisation sont

$$
\vec{M}=\frac{\vec{B}}{\mu_{0}}=\vec{E}^{\star}, \quad \vec{P}=-\varepsilon_{0} \vec{E}=-\vec{B}^{\star}
$$

En effet, les potentiels de polarisation (1) s'écrivent alors

$$
\overrightarrow{\mathrm{II}}=-\frac{\varepsilon_{0}}{4 \pi} \int_{\nu}[\vec{E}] \frac{\mathrm{d} v}{r}, \quad \overrightarrow{\boldsymbol{\theta}}=\frac{\mathrm{I}}{4 \pi} \int_{v}[\vec{B}] \frac{\mathrm{d} v}{r} .
$$

Les définitions (2) des potentiels et antipotentiels donnent alors (17) en vertu de (15), (16). En d'autres termes, les charges fictives de polarisation $\mathrm{du}$ vide coïncident avec les charges-courants vrais.

S'il n'y a que des distributions volumiques, le champ est continu dans tout l'espace jusqu'à l'infini où les intégrales de surface s'annulent [form. (3) et (4)]. S'il y a des distributions superficielles, les intégrales de surface de (3), (4) subsistent et coïncident avec (18), d'après (16).

Il faut bien noter que les formules (7) prennent une signification différente dans la théorie de la polarisation du vide; elles deviennent la définition des vecteurs de champ à partir des vecteurs potentiels, soit

$$
\left.\begin{array}{l}
\vec{B}=\mu_{0}\left(\overrightarrow{\operatorname{grad}} V^{\star}+\partial_{t} \overrightarrow{A^{\star}}\right)+\operatorname{rot} \vec{A}, \\
\vec{E}=-\left(\overrightarrow{\operatorname{grad}} V+\partial_{t} \vec{A}\right)+\varepsilon_{0}^{-1} \operatorname{rot} \vec{A}^{\star} .
\end{array}\right\}
$$

Mais comme les antipotentiels sont nuls, en vertu de (15), cela se réduit à (8). On a toujours I au lieu de $\mathrm{I}, \mathrm{I} / 2$, o puisque le volume $v$ comprend tout l'espace. On change aussi la signification des équations (5) qui s'identifient avec les équations de Maxwell, puisque les courants fictifs s'identifient avec les courants vrais. Les définitions (8), (9) deviennent inutiles puisqu'elles sont remplacées par les expressions (21) plus générales. Les relations (10) ne sont plus valables puisqu'elles sont remplacées par (19).

Remarque. - La nullité des antipotentiels $\vec{V}^{*}, \vec{A}^{*}$ est due à l'absence de second membre dans (15) et à l'absence des intégrales de surface puisque le volume $v$ comprenait tout l'espace.

Cependant, on est parfois amené à considérer un volume fini où règne un champ $\vec{E}, \vec{B}$. Les antipotentiels sont alors différents de zéro à cause des intégrales de surface de (4).

Dans ce cas il faut aussi rétablir I, I/2, o au premier membre de (21) suivant que l'on est dans $v$, sur la surface $S$ qui le limite, ou en dehors de $v$. On consultera à ce sujet une Note de l'auteur [4] qui peut être interprétée dans la théorie de la polarisation du vide, en disant que les champs sont nuls en dehors de $v$. En général ceci ne correspond pas à la réalité et constitue un simple artifice de calcul. Quand le champ est effectivement nul en dehors de $v$, les antipotentiels sont toujours nuls. Exemples : ${ }^{\circ}{ }^{\circ}$ Espace compris entre deux sphères concentriques conductrices portant des charges égales et opposées; $\vec{k}^{*}=$ o, car $[\vec{n} \times \vec{E}]=0$, le champ étant normal aux deux surfaces sphériques qui limitent le domaine; $2^{\circ}$ Courant rectiligne d'intensité $I$ entouré d'un courant en sens inverse circulant sur la surface d'un cylindre coaxial et d'intensité totale $-I$. L'induction $\vec{B}$ est nulle à l'extérieur du cylindre, mais $\sigma^{*}=(\vec{n} \cdot \vec{B})=0$, car $\vec{B}$ est tangent à la surface cylindrique.

3. L'hypothèse de la polarisation du vide en présence de polarisation matérielle. - En l'absence de polarisation matérielle, les vecteurs $\vec{E}$, $\vec{B}$ ne différaient des vecteurs $\vec{D}^{*}, \vec{H}^{*}$ que par un facteur multiplicatif constant et les deux définitions (19) de $\vec{M}, \vec{P}$ étaient identiques. Il n'en est plus de même en présence de polarisation matérielle et l'on a deux possibilités suivant que l'on utilise les vecteurs $\vec{E}, \vec{B}$ ou les vecteurs $\vec{B}^{*}, \vec{E}^{*}$ pour décrire les phénomènes.

io Emploi des vecteurs $\vec{E}, \vec{B}$. - Les équations de Maxwell s'écrivent

$$
\left.\begin{array}{rl}
\overrightarrow{\operatorname{rot}} \vec{E}+\partial_{t} \vec{B} & =0, \\
\operatorname{div} \vec{B} & =0 ; \\
\overrightarrow{\operatorname{rot}} \vec{B}-\varepsilon_{0} \mu_{0} \partial_{t} \vec{E} & =\mu_{0}\left(\vec{i}+\overrightarrow{i^{\prime}}\right), \\
\operatorname{div} \vec{E} & =\varepsilon_{0}^{-1}\left(\rho+\rho^{\prime}\right) ;
\end{array}\right\}
$$


$\vec{i}, \rho$ sont les densités vraies et $\vec{i}^{\prime}, \rho^{\prime}$ sont les densités fictives de courants-charges données a priori (polarisation rigide, par exemple).

Ces équations (22), (23) ne sont pas essentiellement différentes de (15), (16). On a donc une solution en supposant que tout l'espace (espace vide, régions contenant les charges, régions contenant la polarisation matérielle) est polarisée, avec

$$
\vec{P}=-\varepsilon_{0} \vec{E}, \quad \vec{M}=\frac{\vec{B}}{\mu_{0}}
$$

Les formules (20), (2), (21) sont encore valables et donnent la solution cherchée. On a encore $V^{*}=\vec{A}^{*}=0$ et

$$
\square \vec{A}=\mu_{0}\left(\vec{i}+\overrightarrow{i^{\prime}}\right), \quad \square V=\varepsilon_{0}^{-1}\left(\rho+\rho^{\prime}\right) .
$$

Éventuellement avec un volume artificiellement limité, au lieu de $V^{*}=\vec{A}^{*}=$ o, on aurait les relations moins restrictives

$$
A^{\star}=0, \quad \square V^{\star}=0
$$

$2^{\circ}$ Emplor des vecteurs $\vec{B}^{*}, \vec{E}^{*}$. - Avec ces vecteurs les équations de Maxwell s'écrivent

$$
\left.\begin{array}{rl}
\overrightarrow{\operatorname{rot}} \vec{E}^{\star}-\partial_{t} \vec{B}^{\star} & =\vec{i} \\
\operatorname{div} \vec{B}^{\star} & =\rho ; \\
\overrightarrow{\operatorname{rot}} \vec{B}^{\star}+\varepsilon_{0} \mu_{0} \partial_{t} \vec{E}^{\star} & =-\varepsilon_{0}{\overrightarrow{i^{\star}}} \\
\operatorname{div} \vec{E}^{\star} & =\frac{\rho^{\star}}{\mu_{0}}
\end{array}\right\}
$$

On obtient une solution de ces équations en admettant qu'en tout point de l'espace on a une densité de polarisation, $\vec{P}, \vec{M}$, égale à

$$
\vec{P}=-\vec{B}^{\star}, \quad \vec{M}=\vec{E}^{\star}
$$

D'après (1), les potentiels de polarisation $\vec{I}$, $\vec{\Theta}$ s'écrivent

$\vec{\Pi}=-\frac{\mathrm{I}}{4 \pi} \iiint\left[\vec{B}^{\star}\right] \frac{\mathrm{d} v}{r}, \quad \overrightarrow{\boldsymbol{\theta}}=\frac{\mu_{0}}{4 \pi} \iiint\left[\vec{E}^{\star}\right] \frac{\mathrm{d} v}{r}$,

les intégrales de volume étant étendues à tout l'espace. Les potentiels et antipotentiels sont toujours définis par (2) et la relation (7) s'écrit

$$
\left.\begin{array}{l}
\vec{E}^{\star}=\left(\overrightarrow{\operatorname{grad}} V^{\star}+\partial_{t} \vec{A}^{\star}\right)+\mu_{0}^{-1} \overrightarrow{\operatorname{rot}} \vec{A}, \\
\overrightarrow{B^{\star}}=-\varepsilon_{0}\left(\overrightarrow{\operatorname{grad}} V+\partial_{t} \vec{A}\right)+\operatorname{rot} \vec{A}^{\star} .
\end{array}\right\}
$$

C'est la définition des vecteurs de champ à partir des vecteurs potentiels. Avec ces champs (29), les équations (26), (27) sont identiquement satisfaites.

Dans les milieux isotropes, on a

$$
\vec{B}^{\star}=\varepsilon \vec{E}, \quad \vec{E}^{\star}=\frac{\vec{B}}{\mu}
$$

et dans le cas du vide $\varepsilon=\varepsilon_{0}, \mu=\mu_{0}$, ce qui redonne (19). En portant (30) dans (27) on voit que l'on a

$$
\vec{P}=-\varepsilon \vec{E}, \quad \vec{M}=\frac{\vec{B}}{\mu}
$$

Dans l'ancienne conception où seule la matière était polarisée, on avait, au lieu de (31)

$$
\vec{P}=\left(\varepsilon-\varepsilon_{0}\right) \vec{E}, \quad \vec{M}=\left(\frac{\mathrm{I}}{\mu_{0}}-\frac{\mathrm{I}}{\mu}\right) \vec{B}
$$

et en faisant $\varepsilon=\varepsilon_{0}, \mu=\mu_{0}$, dans (32), on avait bien $\vec{P}=\vec{M}=\mathrm{o}$ alors qu'avec (31) le vide est aussi un milieu polarisé.

Dans les substances anisotropes (31), se généralise de la manière suivante

$$
P_{u}=-\varepsilon_{u v} E^{v}, \quad M_{u}=\nu_{u v} B^{v} .
$$

les $\nu_{u \nu}$ étant les réciproques des éléments du tenseur de perméabilité $\mu_{u v}$.

4. Ce qu'apporte de nouveau la théorie de polarisation $\mathbf{d u}$ vide. - On pourrait ne pas interpréter les formules précédentes en faisant du vide un milieu polarisé. Il suffirait de dire que les formules (20) et (28) jointes à la définition (2) des potentiels et antipotentiels donne la solution des équations (22), (23) ou des équations (26), (27).

Les solutions sont valables dans un volume $v$ ou dans tout l'espace suivant que les intégrales (20) ou (28) sont étendues à un volume $v$ ou à tout l'espace. Du point de vue purement mathématique, toutes les formules précédentes reposent sur l'identité suivante [5]

$$
\begin{aligned}
& \underset{0}{1 / 2}\} \psi(x, y, z, v t)=\left\{v^{-2} \partial_{t}^{2}-\Delta\right\} \\
& \times \int_{v} \psi(\xi, \eta, \zeta, v t-r) \frac{\mathrm{d} \xi \mathrm{d} \eta \mathrm{d} \zeta}{4 \pi r},
\end{aligned}
$$

où $\psi$ est une fonction quelconque. On a $\mathrm{I}, \mathrm{I} / 2$, o suivant que le point $x, y, z$ est à l'intérieur de $v$, sur la surface $S$ qui le limite ou en dehors de $v$.

Les formules telles que (31) donnent une interprétation physique de cette identité et conduisent à une théorie du champ qui se présente de la même manière dans le vide et dans les milieux matériels. C'est ainsi que l'on est amené à définir dans les deux cas un potentiel de polarisation ayant un véritable sens physique. Dans les paragraphes suivants nous 
allons calculer ce potentiel pour une charge ponctuelle et pour un courant linéaire, et l'on sait que ces éléments de base servent à construire tous les autres systèmes.

Il ne faut pas confondre ces potentiels qui ont la symétrie de la source considérée avec les potentiels de polarisation que l'auteur a donné dans deux autres publications [1], [2]. Les potentiels de ces publications n'étaient pas, en fait, les potentiels de polarisation de la charge ponctuelle ou du courant linéaire, mais les potentiels de polarisation du système associé (courbe polarisée longitudinalement et issue de la charge; feuillet polarisé normalement et s'appuyant sur le courant); ils ne constituaient donc que des artifices de calcul.

Enfin l'hypothèse de la polarisation du vide a comme conséquence immédiate la nullité de la charge totale de l'Univers. Cette hypothèse s'impose avec moins de force dans l'ancienne conception, quoique l'on admette implicitement que les lignes de force issues d'une charge ponctuelle sont neutralisées par des charges situées sur la sphère concentrique de rayon infini.

5. Potentiel de polarisation d'une charge ponctuelle $q$ au repos (électrostatique). - Il

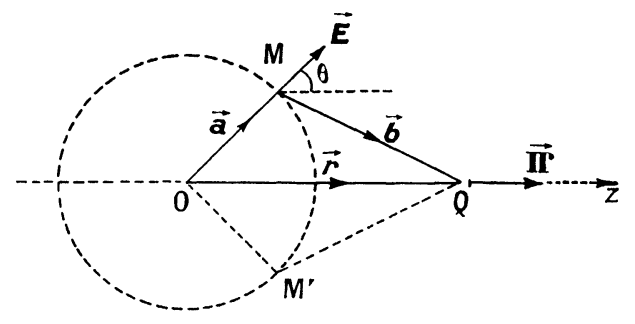

Fig. I.

est donné par (20) où le champ $\vec{E}$ est le champ électrique d'une charge ponctuelle $q$ placée en $O$ (fig. I). Au point $\mathrm{M}$, à une distance $a$ de $\mathrm{O}$, ce champ est égal à

$$
\vec{E}=\frac{q}{4 \pi \varepsilon_{0}} \frac{\vec{a}}{a^{3}}
$$

Si l'on calcule le potentiel de polarisation II au point $\mathrm{Q}$, à une distance $b$ de $\mathrm{M}$ et à une distance $r$ de $\mathrm{O}$, on voit, par raison de symétrie, que seule intervient la composante de $\mathrm{E}$ suivant $\mathrm{O} z$; $\vec{\Pi}$ est donc dirigé suivant $\mathrm{O} z$ et l'on a

$$
\Pi_{z}=-\frac{\varepsilon_{0}}{4 \pi} \frac{q}{4 \pi \varepsilon_{0}} \iiint \frac{\cos \theta}{a^{2}} \frac{\mathrm{d} v}{b},
$$

l'intégrale étant étendue à tout l'espace. On a les relations

$b^{2}=r^{2}+a^{2}-2 a r \cos \theta, \quad$ d'où $\quad b \mathrm{~d} b=\frac{r}{2 \pi a} \mathrm{~d} S$

en désignant par $\mathrm{d} S$ l'élément d'aire de la sphère de rayon $a$, soit

$$
\mathrm{d} S=2 \pi a^{2} \sin \theta \mathrm{d} 0 .
$$

En portant les expressions (37) dans (36), il vient

$$
\begin{aligned}
\Pi_{z} & =-\frac{q}{\mathrm{I} 6 \pi^{2}} \int_{0}^{\infty} \frac{\mathrm{d} a}{a^{2}} \int_{s} \frac{\cos \theta}{b} \mathrm{~d} S \\
& =-\frac{q}{8 \pi r} \int_{0}^{\infty} \frac{\mathrm{d} a}{a} \int \cos \theta \mathrm{d} b \\
& =\frac{q}{\mathrm{I} 6 \pi r^{2}} \int_{0}^{\infty} \frac{\mathrm{d} a}{a^{2}} \int\left(b^{2}-r^{2}-a^{2}\right) \mathrm{d} b .
\end{aligned}
$$

Il faut décomposer en deux le volume d'intégration, avec $a<r$ ou $a<r$. Dans le premier cas, $b$ varie de $(r-a)$ à $(r+a)$ et dans le deuxième $b$ varie de $(a-r)$ à $(a+r)$, d'où

$$
\begin{aligned}
\mathrm{II}_{z}=\frac{q}{\mathrm{I} 6 \pi r^{2}}\{ & \int_{r}^{r} \frac{\mathrm{d} a}{a^{2}} \int_{r-a}^{r+a}\left(b^{2}-r^{2}-a^{2}\right) \mathrm{d} b \\
& \left.+\int_{r}^{\infty} \frac{\mathrm{d} a}{a^{2}} \int_{a-r}^{a-r}\left(b^{2}-r^{2}-a^{2}\right) \mathrm{d} b\right\} .
\end{aligned}
$$

Un calcul élémentaire conduit au résultat suivant

$$
\Pi_{s}=-\frac{q}{8 \pi} .
$$

Par raison de symétrie, on trouverait le même résultat quel que soit le point $Q$; on a alors $\Pi_{r}$ au lieu de $\Pi_{s}$. Le vecteur $\vec{I}$ est donc défini par

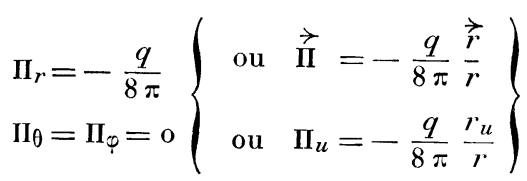

ou enfin

$$
\vec{\Pi}=-\frac{q}{8 \pi} \overrightarrow{\operatorname{grad}} r
$$

De $\overrightarrow{I I}$ on passe aux potentiels et antipotentiels par les formules (2) qui s'écrivent ici simplement

$$
\varepsilon_{0} V=-\operatorname{div} \vec{\Pi}, \quad A^{\star}=-\operatorname{rot} \vec{\Pi} .
$$

Comme le rotationnel d'un gradient est toujours nul, on a

$$
\overrightarrow{A^{\star}}=\mathrm{o}
$$

et pour $V$ l'expression (43) donne bien le potentiel coulombien

$$
V=\frac{q}{4 \pi \varepsilon_{0}} \frac{1}{r} .
$$


$N^{0} 4$. L'HYPOTHĖSE DE LA POLARISATION DU

Pour une distribution volumique de charges dans un volume $v$, en faisant la somme de potentiels tels que (42), on obtient

$$
\overrightarrow{\mathrm{II}}=-\frac{\mathrm{I}}{8 \pi} \overrightarrow{\operatorname{grad}} \int_{v} \rho r \mathrm{~d} v
$$

$\rho$ étant la densité de charge.

6. Potentiel de polarisation d'une droite indéfinie uniformément chargée. — Si $\lambda$ est la densité linéaire de charge sur la droite, on a

$$
\begin{aligned}
\overrightarrow{\mathrm{Il}} & =-\frac{\lambda}{8 \pi} \vec{R}[\mathrm{I}-2 \log R] \\
& =-\overrightarrow{\operatorname{grad}}\left\{\frac{R^{2}}{8 \pi}(\mathrm{I}-2 \log R)\right\},
\end{aligned}
$$

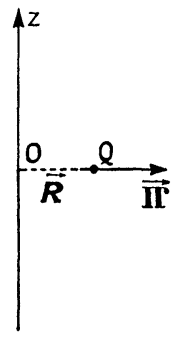

Fig. \&.

où $\vec{R}$ est le vecteur $\overrightarrow{O Q}(f i g .2)$ et $Q$ est le point de coordonnées $x, y$ où l'on calcule le vecteur $\vec{\Pi}$. Les formules (43) donnent

$$
\vec{A}^{\star}=o, \quad V=-\frac{\lambda}{2 \pi \varepsilon_{0}} \log R ;
$$

l'antipotentiel est donc nul et l'on retrouve bien le potentiel logarithmique.

7. Potentiel de polarisation d'un plan uniformément chargé. - Soit $\sigma$ la densité super-

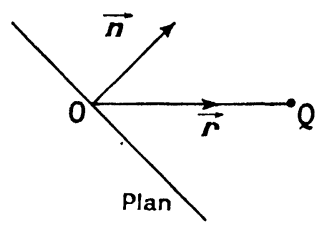

Fig. 3.

ficielle de charge. On suppose que le plan passe par l'origine et il est défini par sa normale positive $\vec{n}$ (fig. 3). On a

$$
\begin{aligned}
\vec{\Pi} & = \pm \frac{\sigma}{20}\left\{\vec{n} r^{2}+2(\vec{n} \cdot \vec{r}) \vec{r}\right\} \\
& = \pm \frac{\sigma}{20} \overrightarrow{\operatorname{grad}}\left\{(\vec{n} \cdot \vec{r}) r^{2}\right\} .
\end{aligned}
$$

Avec (49), les formules (43) donnent

$$
\vec{A}^{\star}=0, \quad V=-( \pm) \frac{\sigma}{2 \varepsilon_{0}}(\vec{n} \cdot \vec{r}) .
$$

Les formules (21) donnent le champ $\vec{E}$, soit

$$
\vec{E}=-\overrightarrow{\operatorname{grad}} V= \pm \frac{\sigma}{2 \varepsilon_{0}} \vec{n}
$$

8. Potentiel de polarisation du dipôle électrique pur. - On l'obtient par un passage à la limite à partir du potentiel de polarisation de deux charges ponctuelles $q,-q($ fig. 4$)$. On fait tendre $A_{1}$ et $A_{2}$ vers zéro; donc $r_{1} \rightarrow r, r_{2} \rightarrow r ; \theta_{1} \rightarrow \theta_{2} \rightarrow \theta$. Dans

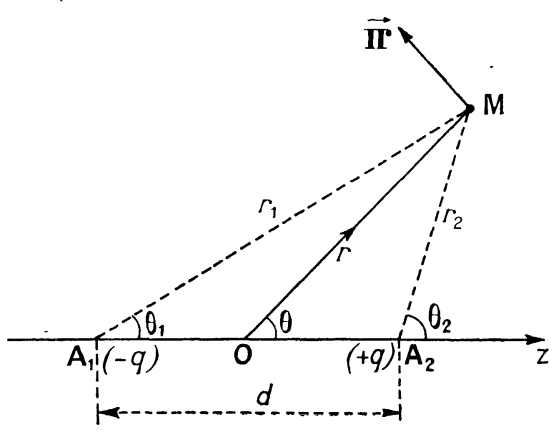

Fig. 4.

ce passage à la limite on maintient le produit $q d$ constamment égal à $p$ malgré que $q \rightarrow \infty, d \rightarrow 0$, d'où

$$
q\left(r_{2}-r_{1}\right) \not \#-q d \cos \theta_{1} \rightarrow-p \cos \theta
$$

de (42), on déduit

$$
\vec{\Pi}=-\frac{q}{8 \pi} \overrightarrow{\operatorname{grad}}\left(r_{2}-r_{1}\right) \rightarrow \frac{p}{8 \pi} \overrightarrow{\operatorname{grad}}(\cos \theta) .
$$

On peut donc écrire

$$
\overrightarrow{\mathrm{II}}=\frac{p}{8 \pi} \overrightarrow{\operatorname{grad}}\left(\vec{p} \cdot \frac{\vec{r}}{r}\right) .
$$

En coordonnées polaires, les composantes de $\vec{I}$ suivant les $r, \varphi$ et $\theta$ croissants, sont

$$
\left.\begin{array}{c}
\Pi_{\varphi}=\Pi_{r}=0, \\
\Pi_{\theta}=\frac{p}{8 \pi r} \frac{\partial}{\partial \theta}(\cos \theta)=-\frac{p \sin \theta}{8 \pi r} \cdot
\end{array}\right\}
$$

Comme c'est un gradient, on a toujours

$$
\overrightarrow{A^{\star}}=-\overrightarrow{\operatorname{rot}} \overrightarrow{I I}=0
$$

et, d'après l'expression de la divergence en coordonnées polaires, on a

$$
\varepsilon_{0} V=-\operatorname{div} \vec{I}=-\frac{\mathrm{I}}{r \sin \theta} \frac{\partial}{\partial \theta}\left(\sin \theta \Pi_{\theta}\right)=\frac{p \cos \theta}{4 \pi r^{2}} .
$$


On retrouve ainsi l'expression classique du potentiel d'un dipôle ponctuel. On comparera le potentiel de polarisation (54) avec le potentiel de polarisation classique qui est différent [6]. Ce dernier donne un antipotentiel $\vec{A}^{*}$ non nul; on en conclut que le potentiel classique n'est pas le potentiel du dipôle électrique pur, mais qu'il est aussi le potentiel de polarisation du tourbillon magnétique élémentaire.

9. Potentiel de polarisation d'un courant linéaire fermé (magnétostatique). - Soit I l'intensité du courant qui circule dans la courbe $\mathrm{C}$ (fig. 5). Le potentiel de polarisation $\vec{\theta}$ est donné par la formule (20). Quand on remplace B par son expression au point $Q$,

$$
\vec{B}=\frac{\mu_{0} I}{1 \pi} \int_{C}\left[\overrightarrow{\mathrm{d} \iota} \times \frac{\vec{a}}{a^{3}}\right]
$$

on obtient

$$
\overrightarrow{\boldsymbol{\theta}}=\frac{\mu_{0} I}{\mathrm{I} 6 \pi^{2}} \int_{C}\left[\overrightarrow{\mathrm{d} l} \times \iiint^{\cdot \frac{\vec{a}}{a^{3}}} \frac{\mathrm{d} v}{b}\right] .
$$

Nous avons montré dans un paragraphe précédent, que nous avions

$$
\iint \frac{\vec{a}}{a^{3}} \frac{\mathrm{d} v}{b}=2 \pi \frac{\vec{r}}{r}=2 \pi \overrightarrow{\operatorname{grad}} r
$$

l'intégrale triple étant étendue à tout l'espace;

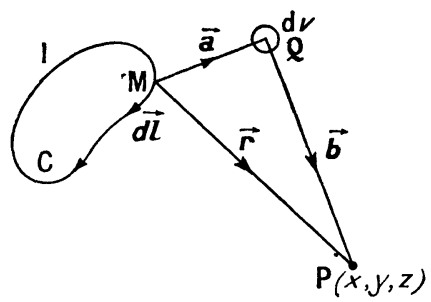

Fig. 5.

en portant cette expression (60) dans (59), il vient

$$
\vec{\Theta}=\frac{\mu_{0} I}{8 \pi} \int_{C}[\overrightarrow{\mathrm{d} l} \times \overrightarrow{\operatorname{grad}} r]=-\overrightarrow{\operatorname{rot}}\left\{\frac{\mu_{0} I}{8 \pi} \int_{C} r \overrightarrow{\mathrm{d} l}\right\}
$$

Les formules (2) permettent de calculer les potentiels et antipotentiels à partir de (61); comme la divergence d'un rotationnel est toujours nulle, on a

$$
V^{\star}=-\operatorname{div} \overrightarrow{\boldsymbol{\theta}}=0 .
$$

Pour $\vec{A}$, après avoir utilisé l'identité

$$
\overrightarrow{\text { rot }} \overrightarrow{\text { rot }}=\overrightarrow{\text { grad }} \operatorname{div}-\Delta,
$$

on obtient

$$
\vec{A}=-\overrightarrow{\operatorname{grad}}\left\{\frac{\mu_{0} I}{8 \pi} \int_{C}(\overrightarrow{\mathrm{d} l} \operatorname{\operatorname {grad}} r)\right\}+\frac{\mu_{0} I}{8 \pi} \int_{C} \Delta r \overrightarrow{\mathrm{d} l} .
$$

Dans la première intégrale de (63), on peut remplacer gradient de $r$ par moins gradient primé de $r$ (gradient par rapport aux coordonnées de M) et transformer en intégrale de surface ( $S$ étant une surface s'appuyant sur C), soit

$$
\int_{C} \overrightarrow{\mathrm{d} l} \overrightarrow{\operatorname{grad}^{\prime}} r=\int_{S}\left(\vec{n} \overrightarrow{\operatorname{rot}}^{\prime} \overrightarrow{\operatorname{grad}}^{\prime} r\right) \mathrm{d} S=0,
$$

car le rotationnel d'un gradient est toujours nul.

Comme $\Delta r=\frac{2}{r}$, il reste l'expression classique du potentiel-vecteur

$$
\vec{A}=\frac{\mu_{0} I}{4 \pi} \int_{C} \frac{\overrightarrow{\mathrm{d} l}}{r} .
$$

Pour une distribution volumique de courants, on applique la formule (61) à chaque tube de courant élémentaire; en remplaçant $I$ par $\mathrm{d} I$, puis en faisant la somme pour tous les tubes de courants élémentaires, on a

$$
\overrightarrow{\boldsymbol{\theta}}=-\overrightarrow{\operatorname{rot}}\left\{\frac{\mu_{0}}{8 \pi} \int \mathrm{d} \boldsymbol{l} \int r \overrightarrow{\mathrm{d} l}\right\}
$$

et comme $\mathrm{d} I \overrightarrow{\mathrm{d} l}=\vec{i} \mathrm{~d} v$, il vient en définitive

$$
\overrightarrow{\boldsymbol{\theta}}=-\overrightarrow{\operatorname{rot}}\left\{\frac{\mu_{0}}{8 \pi} \int_{v} r \vec{i} \mathrm{~d} v\right\} \text {. }
$$

10. Potentiel de polarisation du tourbillon électrique pur. - Pour obtenir le tourbillon électrique, on part d'un courant linéaire et l'on fait tendre vers zéro les dimensions de la courbe $\mathrm{C}$, en la laissant semblable à elle-même. Dans ce passage à la limite on maintient constant le moment magnétique du courant $\vec{p}$ défini par $\left({ }^{2}\right)$

$$
\vec{p}=I \int_{S} \vec{n} \mathrm{~d} S=\frac{I}{2} \int_{C}[\vec{r} \times \overrightarrow{\mathrm{d} l}] .
$$

Le potentiel de polarisation du courant fini est donné par (61); on peut écrire successivement

$$
\begin{aligned}
\int_{C} r \overrightarrow{\mathrm{d} l} & =\int_{S}\left[\vec{n} \times \overrightarrow{\operatorname{grad}^{\prime}} r\right] \mathrm{d} S \\
& =-\int_{S}\left[\vec{n} \times \overrightarrow{\operatorname{grad}^{2}} r\right] \mathrm{d} S .
\end{aligned}
$$

Quand les dimensions du courant tendent vers

(2) Voir, par exemple : E. Durand, Électrostatique et Magnétostatique, Masson, Paris, r 953 , p. 482 . 
zéro, on peut faire sortir $\overrightarrow{\text { grad }} r$ de l'intégrale, d'où

$$
\vec{\Theta}=\overrightarrow{\operatorname{rot}}\left\{\frac{\mu_{0}}{8 \pi}[\vec{p} \times \overrightarrow{\operatorname{grad}} r]\right\}
$$

c'est le résultat cherché.

Les potentiels et antipotentiels se calculent à partir de (70) par les formules (2). Comme la divergence d'un rotationnel est toujours nulle, on a

$$
V^{\star}=-\operatorname{div} \vec{\theta}=0
$$

Avant de calculer le potentiel-vecteur $\vec{A}$, écrivons d'abord le potentiel de polarisation sous la forme

$$
\begin{aligned}
\vec{\Theta} & =-\frac{\mu_{0}}{8 \pi} \overrightarrow{\operatorname{rot}} \overrightarrow{\operatorname{rot}}(\vec{p} \cdot r) \\
& =-\frac{\mu_{0}}{8 \pi} \overrightarrow{\operatorname{grad}}\{\vec{p} \cdot \overrightarrow{\operatorname{grad}} r\}+\frac{\mu_{0}}{8 \pi} \Delta r \\
& =-\overrightarrow{\operatorname{grad}}\left\{\frac{\mu_{0}(\vec{p} \cdot \vec{r})}{8 \pi r}\right\}+\frac{\mu_{0}}{4 \pi r} \vec{p} .
\end{aligned}
$$

Comme le rotationnel d'un gradient est toujours nul, on a

$$
\vec{A}=\overrightarrow{\operatorname{rot}} \vec{\Theta}=\frac{\mu_{0}}{8 \mid \pi}\left[\overrightarrow{\operatorname{grad}}\left(\frac{\mathrm{I}}{r}\right) \times \vec{p}\right]=\frac{\mu_{0}}{4 \pi}\left[\vec{p} \times \frac{\vec{r}}{r^{3}}\right]
$$

C'est bien la valeur classique (voir, par exemple, l'Ouvrage déjà cité de l'auteur, p. 520).

On peut expliciter l'expression (72) et écrire

$\vec{\Theta}=\frac{\mu_{0}}{8 \pi}\left\{\frac{\vec{p}}{r}+(\vec{p} \cdot \vec{r}) \frac{\vec{r}}{r^{3}}\right\}=r_{v}\left(p^{u} r^{v}+p^{v} r^{u}\right) \frac{\mu_{0}}{8 \pi r^{3}}$.

Quand on compare le potentiel de polarisation (74) au potentiel de polarisation classique, on voit qu'il $\mathrm{y}$ a en moins le terme qui donnerait un $V^{*}$ non nul et qui correspondrait au dipôle magnétique; ici, (71), (73) ne contiennent pas cet antipotentiel $V^{*}$ du dipôle magnétique et c'est pourquoi nous l'appelons le tourbillon électrique pur : on peut dire que l'autre potentiel de polarisation correspond à la source mixte qui comprend à la fois le tourbillon électrique et le dipôle magnétique.

11. Les potentiels de polarisation d'une charge ponctuelle en mouvement $(*)$. - Voici l'expression de ces potentiels

$$
\begin{aligned}
& \overrightarrow{\boldsymbol{I}}=\frac{q}{8 \pi}\left\{\frac{\vec{\alpha}-\vec{\beta}}{(\vec{\alpha} \cdot \vec{\beta})-\mathrm{I}}\right\}, \\
& \overrightarrow{\boldsymbol{\theta}}=\frac{q c \mu_{0}}{8 \pi}\left\{\frac{[\vec{\alpha} \times \vec{\beta}]}{(\vec{\alpha} \cdot \vec{\beta})-\mathrm{I}}\right\}_{\tau},
\end{aligned}
$$

(*) Nole ajoutée à la correction des épreuves. - Dans une publication récente [8], l'auteur a montré que ces deux vecteurs $\overrightarrow{I I}$ et $\vec{\theta}$ pourraient se déduire d'un quadrivecteur unique très simple. où $\vec{\alpha}=\frac{\vec{r}}{r}$ et $\vec{\beta}=\frac{\vec{v}}{c} ; \vec{v}$ est la vitesse de la particule et l'indice $\tau$ indique que toutes ces expressions doivent être prises au temps retardé. Pour justifier cette forme (75), (76) des potentiels de polarisation nous allons montrer que les formules (2) de définitions des potentiels ordinaires conduisent aux potentiels de Liénard-Wiechert.

Pour effectuer ces calculs on notera que l'on a, en désignant par $\gamma^{u}$ l'accélération retardée

$$
\left.\begin{array}{c}
\partial_{u} \tau=\frac{\mathbf{I}}{c} \frac{\alpha_{u}}{[(\vec{\alpha} \cdot \vec{\beta})-\mathrm{I}]}, \quad \partial^{u} \beta v=\frac{\mathbf{I}}{c^{2}} \frac{\alpha^{u} \gamma^{v}}{[(\vec{\alpha} \cdot \vec{\beta})-\mathrm{I}]}, \\
\partial u \alpha^{v}=\frac{\alpha^{u} \alpha^{v}-\alpha^{u} \beta^{v}+\delta u v[(\vec{\alpha} \cdot \vec{\beta})-\mathrm{I}]}{r[(\vec{\alpha} \cdot \vec{\beta})-\mathrm{I}]} ; \\
\partial_{\iota} \tau=-\frac{\mathrm{I}}{[(\vec{\alpha} \cdot \vec{\beta})-\mathrm{I}]}, \quad \partial_{t} \beta u=-\frac{\gamma^{u}}{[(\vec{\alpha} \cdot \vec{\beta})-\mathrm{I}]}, \\
\partial_{t} \alpha^{u}=c \frac{\left[\beta^{u}-\alpha^{u}(\vec{\alpha} \cdot \vec{\beta})\right.}{r[(\vec{\alpha} \cdot \vec{\beta})-\mathrm{I}]} .
\end{array}\right\}
$$

Toutes ces formules doivent être prises au temps retardé $\tau$. A l'aide de ces expressions ou en prenant leur contraction $\partial_{\nu} \alpha^{\nu}$ ou $\partial_{v} \beta^{\nu}$ on trouve, après un calcul élémentaire mais assez laborieux, les relations

$$
V=\frac{q}{4 \pi \varepsilon_{0}}\left\{\frac{\mathrm{I}}{r \Delta}\right\}_{\tau}, \quad \vec{A}=\frac{q c \mu_{0}}{4 \pi}\left\{\frac{\vec{\beta}}{r \Delta}\right\}_{\tau},
$$

avec

$$
\left.\begin{array}{c}
\Delta=\mathrm{I}-(\vec{\alpha} \cdot \vec{\beta}), \quad c=\frac{\mathrm{I}}{\sqrt{\varepsilon_{0} \mu_{0}}}, \\
\left.c \mu_{0}=\sqrt{\frac{\mu_{0}}{\varepsilon_{0}}}=Z_{0} \quad \text { impédance du vide }\right) .
\end{array}\right\}
$$

On reconnaît les potentiels de Liénard-Wiechert et c'est bien ce que nous voulions obtenir.

On trouve, de même,

$$
\overrightarrow{A^{\star}}=\varepsilon_{0} \partial_{l} \vec{\theta}-\overrightarrow{\operatorname{rot}} \overrightarrow{I I}=0, \quad \mu_{0} V^{\star}=-\operatorname{div} \vec{\theta}=0
$$

c'est-à-dire que les antipotentiels sont nuls.

Quand $\vec{v}$ tend vers zéro, le potentiel (75) redonne l'expression (42) que nous avons trouvée en électrostatique par intégration du champ coulombien.

De (75), (76), on tire aisément les relations

$$
c \mu_{0}[\overrightarrow{\mathrm{I}} \times \vec{\beta}]=\overrightarrow{\boldsymbol{\theta}}\left\{\begin{array}{l}
(\overrightarrow{\mathrm{II}} \cdot \overrightarrow{\boldsymbol{\theta}})=0, \\
(\vec{\beta} . \overrightarrow{\boldsymbol{\theta}})=0 ; \\
(\vec{\alpha} . \overrightarrow{\mathrm{II}})=-\frac{q}{8 \pi} \cdot
\end{array}\right\}
$$


La figure 6 indique les dispositions relatives des différents vecteurs. Quand $\vec{\beta}$ tend vers zéro, le vecteur $\overrightarrow{\text { II }}$ a même direction que $\vec{\alpha}$ et il s'en écarte d'autant plus que $\vec{\beta}$ est plus grand. Le vecteur $\vec{\theta}$ est normal au plan $[\vec{\beta}][\vec{k}]$ et est représenté sur la figure par un point noir entouré d'un cercle. On

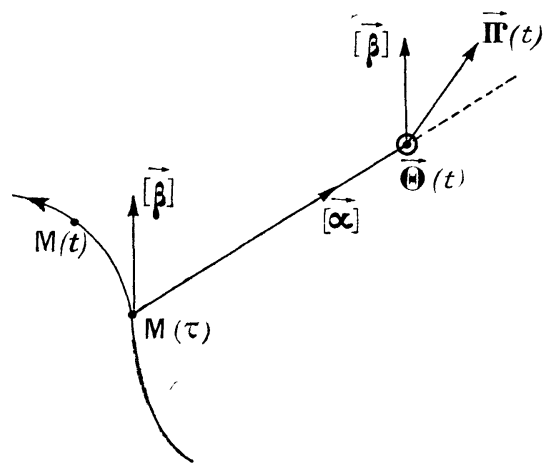

Fig. 6.

notera aussi que les potentiels (75), (76) ne dépendent pas de la distance du point considéré à $M(\tau)$, mais ne font intervenir que le vecteur $\vec{\alpha}$ qui est un vecteur de module unité. On pourrait les appeler les potentiels angulaires.

12. Les potentiels de polarisation d'une distribution volumique de charges et de courants, dépendant du temps. - Si $\rho(x, y, z, t)$ et $\vec{i}(x, y$, $z, t)$ sont les densités de charges et de courants et en plaçant entre crochets les grandeurs retardées

$$
[\uparrow]=p\left(x, y, z, t-\frac{r}{c}\right), \quad[\vec{i}]=\vec{i}\left(x, y, z, t-\frac{r}{c}\right),
$$

ces potentiels ont pour expression

$$
\left.\begin{array}{l}
\vec{\Pi}=-\frac{1}{8 \pi c} \int\{c \rho \vec{\alpha}-[\vec{i}]\} \mathrm{d} v, \\
\vec{\Theta}=-\frac{\mu_{0}}{8 \pi} \int\{\vec{\alpha} \times[\vec{i}]\} \mathrm{d} v,
\end{array}\right\}
$$

On passera des expressions (82) aux expressions (75), (76) en utilisant le jacobien de la transformation qui fait passer du volume retardé au volume actuel pour un système en mouvement [7], et en tenant compte de $\vec{i}=\rho \vec{v}$.

Quand $\rho=$ o et que $\vec{i}$ ne dépend pas du temps, $[\vec{i}]=\vec{i}$ et l'on a

$$
\overrightarrow{I I}=\frac{\mathrm{I}}{8 \pi c} \int_{v}^{\vec{i}} \mathrm{~d} v=0
$$

puisque les lignes de courants sont fermées et tangentes à la surface $S$ qui limite le volume $\boldsymbol{v}$.

L'autre potentiel (82) s'écrit

$$
\vec{\Theta}=-\frac{\mu_{0}}{8 \pi} \overrightarrow{\operatorname{rot}} \int_{v} \vec{i} r \mathrm{~d} v .
$$

On retrouve ainsi, comme il se doit, la formule (67). Enfin, si $\vec{i}=0$ on retrouve (46).

Manuscrit reçu le 6 juillet 1954 .

BIBLIOGRAPHIE.

[1] Durand E. - Le champ $\vec{H}$ et l'induction $\vec{B}$ d'un courant linéaire dans le vide. C. R. Acad. Sc., I954, 238, 1394 .

[2] Durand E. - Le champ $\vec{E}$ et l'induction $\vec{D}$ d'une charge ponctuelle dans le vide. C. R. Acad. Sc., r 954, 238, 1478 .

[3] Durand E. - Sur les formules générales de la théorie des milieux polarisés. C. R. Acad. Sc., r $954,238,2504$.

[4] Durand E. - Solutions générales des équations de Maxwell. C. R. Acad. Sc., 1953, 236, 1407.

[5] Durand E. - Identités conduisant aux solutions des équations aux dérivées partielles linéaires et à coefficients constants. Bull. Soc. Math. Fr., I 954, 82, 36 r-4 I I.
[6] Durand E. - Les distributions de dipôles. Ann. Phys. I $954,9,493-523$.

[7] Durand E. - Passage de l'intégrale des potentiels retardés aux formules de Liénard-Wiechert. $C$. $R$. Acad. Sc., 1946, 222, 284.

[8] Durand E. - Sur la possibilité de considérer les potentiels et les champs comme des grandeurs densitaires; nouveau type de quadrivecteur. C. R. Acad. Sc., 1954, 329, 75 r. 